\title{
Comparación entre el índice de masa corporal y el índice de Control Nutricional para determinar la gravedad en pacientes con sepsis abdominal
}

\author{
Comparison between the body mass index and the Controlling Nutritional status to \\ determine the severity in patients with abdominal sepsis
}

\author{
Ansony R. Godinez-Vidal'* Elvira Guzmán-Valencia², Jorge L. De León-Rendón ${ }^{3}$, \\ Mariel González-Calatayud4, Sandra C. López-Romero y Noé I. Gracida-Mancilla ${ }^{4}$ \\ ${ }^{1}$ Departamento de Cirugía General; '2Servicio de Nutrición, Departamento de Cirugía General; ${ }^{3}$ Departamento de Cirugía de Colon, Recto y Ano; \\ ${ }^{4}$ Clínica de Sepsis Abdominal, Departamento de Cirugía General. Hospital General de México Dr. Eduardo Liceaga, Ciudad de México, México
}

\begin{abstract}
Resumen
Introducción: La respuesta sistémica del organismo, en defensa ante el agente agresor, genera una respuesta catabólica aguda que conduce a deterioro del estado nutricional. Objetivo: Comparar la utilidad del índice de masa corporal (IMC) y del índice de Control Nutricional (CONUT) para determinar la gravedad en pacientes con sepsis abdominal. Método: Estudio retrospectivo, descriptivo, transversal, en pacientes con diagnóstico de sepsis abdominal, de abril de 2016 a febrero de 2017. Resultados: Se incluyeron 153 casos (61 mujeres y 92 hombres). El principal órgano causante de sepsis abdominal fue el apéndice (43\%). La mortalidad fue del 15\%. El IMC promedio fue de 27.31. El CONUT promedio fue de 5.5, Los hallazgos, sometidos a la prueba $U$ de Mann-Whitney, mostraron al evaluar el IMC contra la escala SOFA (Sequential Organ Failure Assessment Score) una $p=0.025$; no se encontró significancia al evaluar el IMC contra APACHE II (Acute Physiology and Chronic Health Evaluation) $(p=0.322)$ ni contra la mortalidad $(p=0.646)$. En cuanto a CONUT, se encontró significancia al compararla contra APACHE II, SOFA y la mortalidad ( $p=0.002, p=0.001$ y $p=0.007$, respectivamente). Conclusión: EI grado de malnutrición determinado por CONUT se relaciona con la gravedad determinada mediante APACHE II y SOFA, $y$ con la mortalidad. EI IMC no se relaciona con la gravedad por APACHE II ni con la mortalidad, aunque sí parece relacionarse con la gravedad evaluada mediante SOFA.
\end{abstract}

PALABRAS CLAVE: Gravedad. Mortalidad. Nutrición. Sepsis.

\begin{abstract}
Background: The systemic response of the organism, in defense against the aggressor agent, generates acute catabolic response, which leads to deterioration of the nutritional status. Objective: Compare the usefulness of the body mass index (BMI) and the CONUT scale to determine the severity in abdominal sepsis. Methods: Retrospective, descriptive, cross-sectional study in patients with diagnosis of abdominal sepsis, from April 2016 to February 2017. Results: We included 153 cases (61 female and 92 male); mean age of 47.44 years, the main organ causing abdominal sepsis was the appendix $43 \%$. Mortality of $15 \%$. An average BMI of 27.31, CONUT score of 5.5, was obtained. The findings, subjected to the Mann-Whitney $u$ test, showed statistical significance when evaluating BMI against SOFA $(p=0.025)$; no significance was found when evaluating the BMI against APACHE II ( $p=0.322)$, nor against mortality $(p=0.646)$. Regarding CONUT, significance was found
\end{abstract}

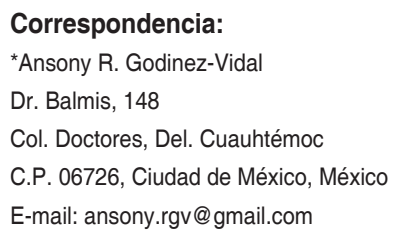

Fecha de recepción: 12-04-2018

Fecha de aceptación: 16-06-2018 DOI: $10.24875 / C I R U .18000318$
Cir Cir. 2019;87:605-610 www.cirugiaycirujanos.com 
when comparing against APACHE II, SOFA and mortality ( $p=0.002, p=0.001$ and $p=0.007$, respectively). Conclusions: The level of malnutrition determined by CONUT is related to the severity determined by APACHE II, SOFA and mortality. BMI is not related to severity by APACHE II or mortality; although it does seem to relate to the severity evaluated by the SOFA scale.

KEY WORDS: Severity. Mortality. Nutrition. Sepsis.

\section{Introducción}

La peritonitis secundaria es frecuente en la práctica quirúrgica general' ${ }^{1}$. Resulta sumamente difícil hacer una estimación de la incidencia anual de la sepsis abdominal por la gama tan amplia de patologías que pueden llegar a desarrollarla (apendicitis, úlceras gástricas, enfermedad diverticular complicada, etc.). Dicha diversidad en enfermedades hace que el abordaje quirúrgico y terapéutico carezca de uniformidad².

La respuesta inflamatoria-antiinflamatoria del organismo en defensa ante el agente agresor, con el consiguiente deterioro del estado nutricional, incrementa la mortalidad ${ }^{3,4}$. El índice de masa corporal (IMC) es el parámetro sugerido por la Organización Mundial de la Salud (OMS) para valorar el estado nutricional del paciente hospitalizado; sin embargo, la gran actividad metabólica generada podría no verse reflejada en el IMC, ya que la pérdida en peso causada por la depleción proteica podría verse compensada por la retención de líquido de la reacción inflamatoria aguda, lo que obliga a evaluar con más detalle el estado nutricional ${ }^{5}$. En algunos estudios se reporta que cuando el índice de Control Nutricional (CONUT) no se incluye en los análisis estadísticos la obesidad parece ser protectora en las unidades quirúrgicas. Este efecto protector, denominado «la paradoja de la obesidad», ha sido reportado en asociación con múltiples afecciones, incluyendo la infección por el virus de la inmunodeficiencia humana/sida, la enfermedad renal terminal y la insuficiencia cardiaca aguda ${ }^{6-8}$.

Parte del manejo integral del paciente con sepsis abdominal es determinar el índice CONUT, ya que tiene unas altas sensibilidad y especificidad para establecer el grado de desnutrición, y se ha empleado para evaluar la gravedad de diferentes enfermedades, como la neumopatía obstructiva, el cáncer de pulmón, el cáncer de esófago, la enfermedad coronaria, el infarto agudo de miocardio y el cáncer colorrectal $^{9-11}$.

Se ha observado que los pacientes hospitalizados con sepsis abdominal tienen problemas en su estado nutricional, debido a que la infección grave desencadena una inflamación generalizada que origina una respuesta catabólica exagerada, lo que aunado al ayuno prolongado explica dicho deterioro nutricional, pues provoca, en otros aspectos, pérdida del apetito, pérdida de peso, depleción muscular y pérdida de grasa corporal. Este tipo de patología exige que los profesionales de diferentes especialidades (médicos, nutriólogos, psicólogos, etc.) y ámbitos sanitarios estén preparados para abordarla. Dada la urgencia y las condiciones especiales del paciente séptico, hay que contar con herramientas de diagnóstico accesibles en todo momento y fáciles de utilizar.

La presente investigación se propuso como objetivo comparar la utilidad del IMC y del CONUT para determinar la gravedad en pacientes con sepsis abdominal atendidos en la Clínica de la Sepsis Abdominal del Hospital General de México Dr. Eduardo Liceaga.

\section{Método}

Se realizó un estudio observacional, retrospectivo, descriptivo, transversal, de pacientes con diagnóstico de sepsis abdominal por peritonitis secundaria, confirmado mediante cirugía urgente, atendidos en la clínica de sepsis del Hospital General de México Dr. Eduardo Liceaga durante el periodo de abril 2016 a febrero 2017. En todos los casos se determinaron el IMC y el CONUT. Se obtuvo la distribución muestral para cada variable y se encontró una distribución no normal, por lo que se procedió a categorizar ambas variables. Se construyó una curva de sensibilidad-especificidad para el puntaje CONUT como predictor de mortalidad. Se obtuvo un área bajo la curva de 0.665 , con significación estadística $(p=0.012)$, con el mejor nivel de discriminación en un puntaje CONUT de 5 (sensibilidad del $69 \%$ y especificidad del 67\%) para determinar la mortalidad. La curva ROC (Receiver Operating Characteristic) del IMC reportó una curva sin significancia estadística $(p=0.994$, con un AUC (Area Under the Curve) de 0.501). Se asumió el rango de IMC de 19 a 25 referido en la literatura como parámetro de normonutrición, y se consideró malnutrición un IMC $<190>25$. La 
Tabla 1. Al comparar el IMC contra las escalas de gravedad mediante la prueba $U$ de Mann-Whitney se halló significancia estadística contra SOFA ( $p=0.025)$, pero no contra APACHE II $(p=0.322)$ ni contra la mortalidad $(p=0.646)$

\begin{tabular}{lccccc}
\hline Variable & \multicolumn{2}{c}{ Evaluación por IMC } & p & OR \\
\cline { 2 - 4 } & $\begin{array}{c}\text { Malnutridos }>25 \\
\text { Media (DE) }\end{array}$ & $\begin{array}{c}\text { Normonutridos }>18.5 \\
\mathbf{a}<\mathbf{2 4 . 9} \\
\text { Media (DE) }\end{array}$ & & \\
\hline APACHE II & $11.3(6.9)$ & $9.6(5.9)$ & 0.322 & - \\
SOFA & $3.8(2.3)$ & $3.1(1.4)$ & 0.025 & - \\
Mortalidad & $16 / 100(16 \%)$ & $7 / 53(13 \%)$ & 0.646 & - \\
\hline
\end{tabular}

DE: desviación estándar; OR: odds ratio.

gravedad de la sepsis abdominal se determinó mediante dos escalas fisiológicas: APACHE II y SOFA; y también se determinó la mortalidad. Se dividió la muestra en casos leves (APACHE II $<14$, SOFA $<$ 5 , desenlace vivo) y casos graves (APACHE II $>15$, SOFA $>6$, desenlace fallecido). La literatura refiere estos puntos de corte como predictores para un desenlace desfavorable. Por ello se dividió la muestra para determinar si los grupos con mayores puntajes en nuestras escalas de gravedad se relacionan con mayor puntaje en CONUT y con el IMC. Se compararon los grupos empleando tablas de $2 \times$ 2 y se evaluaron las frecuencias. Para determinar la asociación entre el puntaje de IMC y el puntaje CONUT con las escalas de gravedad APACHE II y SOFA, y con la mortalidad, se empleó la prueba de Mann-Whitney.

\section{Resultados}

Se incluyeron 153 casos (61 mujeres y 92 hombres), con una media de edad de 47.77 años (desviación estándar $[D E] \pm 18.37$ ). Los órganos causantes de la sepsis abdominal fueron el apéndice $(43 \%)$, el intestino delgado $(20 \%)$, el colon (15\%), el estómago $(5 \%)$, las vías biliares $(4 \%)$, el útero y sus anexos $(3 \%)$, el páncreas $(2 \%)$, el riñón (1\%) y otros $(3 \%)$. Fallecieron 23 pacientes (15\%). Las puntuaciones medias de gravedad para la muestra fueron: APACHE II, 10.79 (DE \pm 6.64$)$; SOFA, 3.58 (DE \pm 1.6$)$. Se obtuvo un IMC promedio para la muestra de $27.31(\mathrm{DE} \pm 4.5)$. El puntaje CONUT promedio fue de $5.5(\mathrm{DE} \pm 3.5)$. Al comparar el IMC contra las escalas de gravedad (Tabla 1) mediante la prueba $U$ de Mann-Whitney se halló significancia estadística al analizar el IMC contra SOFA ( $p=0.025$ ) (Fig. 1); sin embargo, no hubo significancia al evaluar el IMC contra APACHE II



Figura 1. Relación entre el IMC y la escala SOFA.

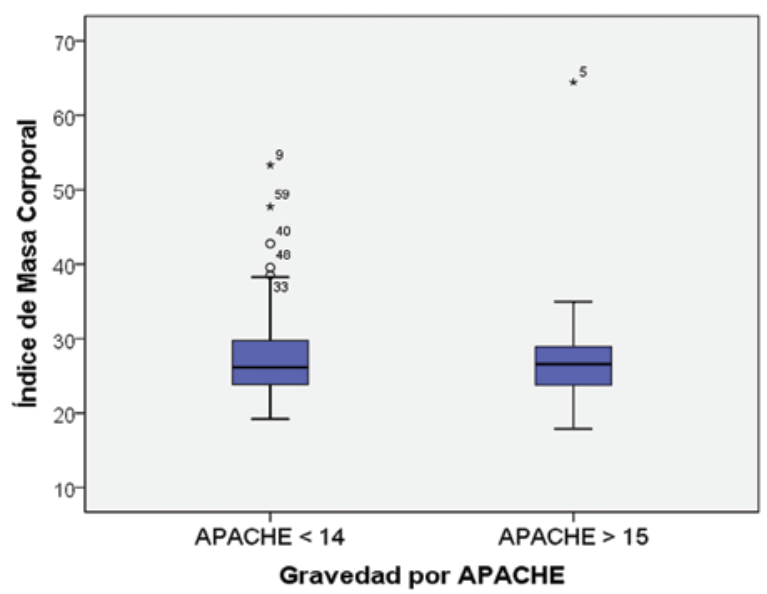

Figura 2. Relación entre el IMC y la escala APACHE II.



Figura 3. Relación entre el IMC y la mortalidad.

$(p=0.322)$ (Fig. 2) ni contra la mortalidad ( $p=0.646)$ (Fig. 3). En cuanto a la comparacion de CONUT contra las escalas de gravedad (Tabla 2), se encontró significancia estadística contra APACHE II (Fig. 4), 
Tabla 2. Al comparar el CONUT contra las escalas de gravedad se encontró significancia estadística contra APACHE II, SOFA y la mortalidad $(p=0.002, p=0.001$ y $p=0.007$, respectivamente)

\begin{tabular}{lcccc}
\hline Variable & \multicolumn{2}{c}{ Evaluación por CONUT } & p & OR \\
\cline { 2 - 3 } & $\begin{array}{c}\text { Malnutridos }>6 \\
\text { Media (DE) }\end{array}$ & $\begin{array}{c}\text { Normonutridos }<5 \\
\text { Media (DE) }\end{array}$ & & \\
\hline APACHE II & $13.2(7.1)$ & $8.8(5.5)$ & 0.002 & 3.44 \\
SOFA & $4.3(2.4)$ & $3.0(1.5)$ & 0.001 & 3.19 \\
Mortalidad & $16 / 67(23.8 \%)$ & $7 / 86(8.1 \%)$ & 0.007 & 0.282 \\
\hline
\end{tabular}

SOFA (Fig. 5) y la mortalidad (Fig. 6) ( $p=0.002$, $p=0.001$ y $p=0.007$, respectivamente).

\section{Discusión}

La malnutrición afecta al 30-50\% de los pacientes hospitalizados de todas las edades, tanto por causas quirúrgicas como médicas, y aumenta a medida que se prolonga la estancia hospitalaria, a pesar de lo cual continúa pasando desapercibida ${ }^{12}$.

Los resultados obtenidos por la investigación indican que predomina el sexo masculino, de acuerdo con lo reportado en la literatura, en la cual el sexo masculino de edad avanzada es más susceptible a padecer sepsis abdominal, debido a que los hombres tienden a desencadenar más rápidamente hipotermia por la existencia previa de una disfunción termorreguladora, y las manifestaciones neurológicas son muy frecuentes ${ }^{13}$.

La causa más común de sepsis abdominal en nuestra serie fue la apendicitis aguda, como se ha mostrado en otros estudios ${ }^{14}$. Reportamos una mortalidad del $15 \%$, que es más baja que la mencionada por Carrillo-Esper, et al. ${ }^{15}$, quienes realizaron un estudio epidemiológico en México, en las unidades de terapia intensiva, y reportaron una mortalidad del $40 \%$; cabe señalar que en su estudio solo incluyeron pacientes con choque séptico. En nuestra serie se obtuvo una media de peso de $72.2 \mathrm{~kg}$, y para la talla, una media de $1.62 \mathrm{~m}$, con una media de IMC de 27.31. Este es un dato muy relevante que puede ser un reflejo de las observaciones epidemiológicas que han señalado que el sobrepeso y la obesidad son cada vez más frecuentes en nuestro país, sobre todo teniendo en mente que la sepsis es una enfermedad con alto consumo calórico y que ocasiona un deterioro nutricional agudo grave. En estudios realizados por la Secretaría de Salud se observa que la población en México no solo tiene

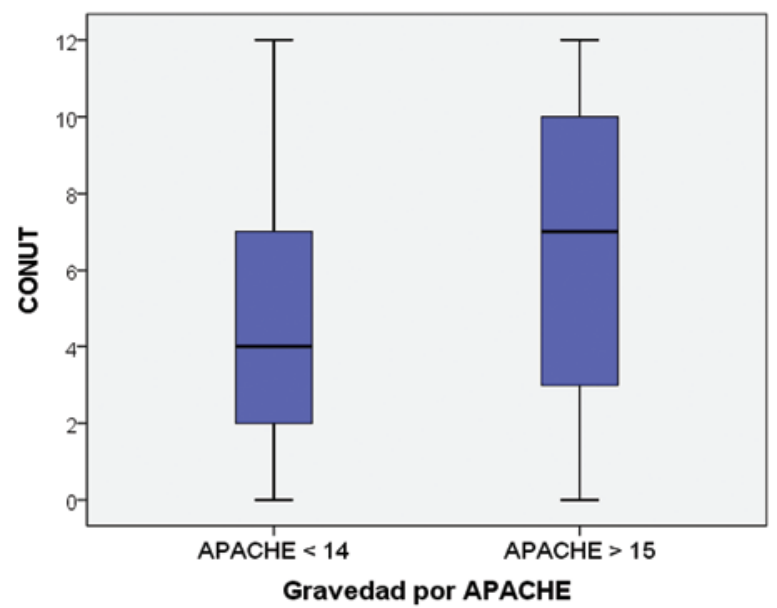

Figura 4. Relación entre el CONUT y la escala APACHE II. En el gráfico se aprecia que, a mayor puntaje CONUT, mayor puntaje APACHE $(p=0.002)$.

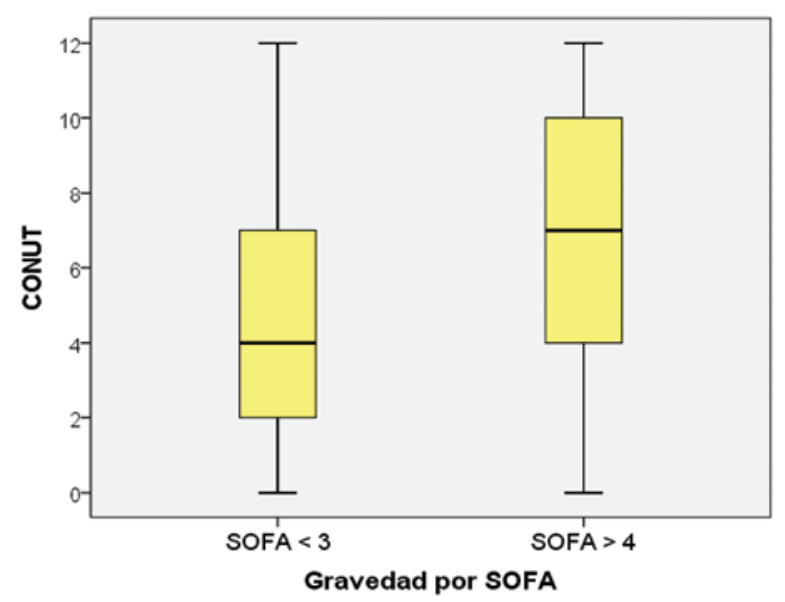

Figura 5. Relación entre el CONUT y la escala SOFA. En el gráfico se aprecia que, a mayor puntaje CONUT, mayor puntaje SOFA $(p=0.001)$.

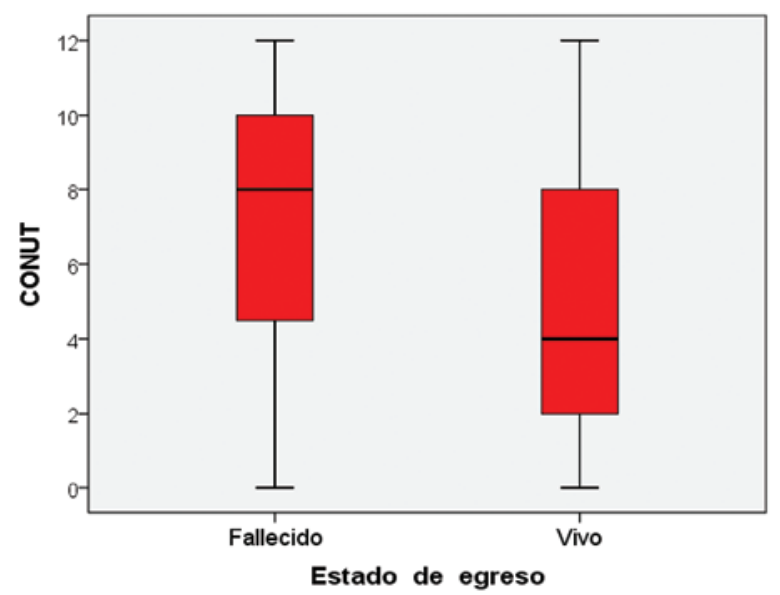

Figura 6. Relación entre el CONUT y la mortalidad. En el gráfico se aprecia que, a mayor puntaje CONUT, mayor mortalidad ( $p=0.007$ ). 
sobrepeso, sino que ha ido en incremento la obesidad, y a pesar de esto la desnutrición continúa estando presente. Tal acontecimiento hizo que se iniciaron reuniones para el establecimiento de la Norma Oficial Mexicana para el manejo de la obesidad $^{16}$.

En los estudios se menciona que el CONUT sirve para conocer el riesgo de que se produzcan cambios en el estado de nutrición en función del diagnóstico, y así poder brindar propuestas de nuevos procedimientos terapéuticos. Se ha empleado para evaluar la gravedad de diferentes enfermedades, como cáncer, trastornos de lípidos crónicos, trastornos hematológicos neoplásicos y trastornos endocrinos crónicos ${ }^{17}$. En la presente investigación se obtuvo un valor medio de CONUT de 5.5. Al evaluar la correlación entre el IMC y el CONUT contra las escalas de gravedad y la mortalidad, debe considerarse que el IMC es un indicador antropométrico, mientras que el CONUT es un indicador bioquímico de desgaste y actividad metabólica. Sugerimos que el IMC refleja, ante todo, el compromiso crónico del paciente, mientras que el CONUT es más un indicador de modificaciones agudas. EI CONUT abarca potencialmente la totalidad de los pacientes registrados en el Sistema Nacional de Salud ${ }^{18}$. En nuestro caso, aunque el CONUT no ha sido validado específicamente para pacientes con sepsis abdominal, encontramos que tiene una sensibilidad del $69 \%$ y una especificad del 60\% para predecir la mortalidad. Es necesaria la detección precoz de la desnutrición por métodos de cribaje aplicados de manera universal a todos los pacientes ingresados en el hospital. Este cribaje tendría utilidad no solo desde el punto de vista clínico, sino también desde el punto de vista bioquímico. Esto es así porque la presencia de malnutrición es un factor pronóstico adverso.

Observamos que el IMC, el cual es el sugerido por la OMS, no se correlaciona con la escala APACHE II (que es la escala mas utilizada en nuestra institución) ni con la mortalidad, por lo que podemos tener una poblacion con sobrepeso y obesidad, y sin embargo con mal estado de nutrición, y sugerimos que el CONUT debería ser una escala necesaria en la valoración del estado nutricional de los pacientes con sepsis abdominal.

\section{Conclusión}

El grado de malnutrición determinado por CONUT se relaciona con la gravedad de la sepsis abdominal determinada mediante APACHE II y SOFA, y con la mortalidad, mientras que el IMC no se relaciona con la gravedad por APACHE II ni con la mortalidad, aunque sí parece relacionarse con la gravedad evaluada mediante la escala SOFA. El índice CONUT es mejor indicador de modificaciones agudas del estado nutricional y de la gravedad en la sepsis abdominal. CONUT no sustituye al IMC, pero debe ser un método de cribaje a realizar desde el ingreso a la institucion.

\section{Financiamiento}

Los autores declaran que no se obtuvo ningún tipo de financiamiento por parte de ninguna empresa.

\section{Conflicto de intereses}

Los autores declaran que no existe ningún conflicto de intereses.

\section{Responsabilidades éticas}

Protección de personas y animales. Los autores declaran que para esta investigación no se han realizado experimentos en seres humanos ni en animales.

Confidencialidad de los datos. Los autores declaran que han seguido los protocolos de su centro de trabajo sobre la publicación de datos de pacientes.

Derecho a la privacidad y consentimiento informado. Los autores han obtenido el consentimiento informado de los pacientes y/o sujetos referidos en el artículo. Este documento obra en poder del autor de correspondencia.

\section{Bibliografía}

1. Anaya $D A$, Nathens $A B$. Risk factors for severe sepsis in secondary peritonitis. Surg Infect (Larchmt). 2003;4:355-62.

2. Cheadle WG, Spain DA. The continuing challenge of intra-abdominal infection. Am J Surg. 2003;186:15S-22S.

3. Lim SL, Ong KC, Chan YH, Loke WC, Ferguson M, Daniels L. Malnutrition and its impact on cost of hospitalization, length of stay, readmission and 3-year mortality. Clin Nutr. 2012;31:345-50.

4. Correia MI, Waitzberg DL. The impact of malnutrition on morbidity, mortality, length of hospital stay and costs evaluated through a multivariate model analysis. Clin Nutr. 2003;22:235-9.

5. WHO Library Cataloguing-in-Publication Data. Global status report on noncommunicable diseases 2010. Geneva: World Health Organization; 2010.

6. Chlebowski RT, Grosvenor M, Lillington L, Sayre J, Beall G. Dietary intake and counseling, weight maintenance, and the course of HIV infection. J Am Diet Assoc. 1995;95:428-32.

7. Kalantar-Zadeh K, Abbott KC, Salahudeen AK, Kilpatrick RD, Horwich TB. Survival advantages of obesity in dialysis patients. Am J Clin Nutr. 2005;81:543-54

8. Fonarow GC, Srikanthan P, Costanzo MR, Cintron GB, Lopatin M; ADHERE Scientific Advisory Committee and Investigators. An obesity paradox in acute heart failure: analysis of body mass index and inhospital mortality for 108,927 patients in the Acute Decompensated Heart Failure National Registry. Am Heart J. 2007;153:74-81.

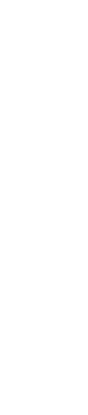


9. Akamine T, Toyokawa G, Matsubara T, Kozuma Y, Haratake N, Takamori S Significance of the preoperative CONUT score in predicting postoperative disease-free and overall survival in patients with lung adenocarcinoma with obstructive lung disease. Anticancer Res. 2017;37:2735-42.

10. Formiga $F$, Chivite D, Corbella X. Utility of the Controlling Nutritiona Status (CONUT) score in patients admitted due to acute heart failure. Int J Cardiol. 2017;235:203.

11. Tokunaga R, Sakamoto Y, Nakagawa S, Ohuchi M, Izumi D, Kosumi K. CONUT: a novel independent predictive score for colorectal cancer patients undergoing potentially curative resection. Int $\mathrm{J}$ Colorectal Dis. 2017;32:99-106.

12. McWhiter JP, Pennington CR. Incidence and recognition of malnutrition in hospital. BMJ. 1994;308:945-8.

13. Taylor RW. Sepsis, sepsis syndrome and septic shock. En: Civetta JM Taylor RW, Kirby RR, editores. Critical care. $2^{\text {nd }}$ ed. Philadelphia: JB Lippincott; 1992. p. 401-6.
14. Schein M, Wittmann DH, Wise L, Condon RE. Abdominal contamination, infection and sepsis: a continnum. Br J Surg. 1997;84:269-72.

15. Carrillo-Esper R, Carrillo-Córdova JR, Carrillo-Córdova LD. Estudio epidemiológico de la sepsis en unidades de terapia intensiva mexicanas. Cir Cir. 2009;77:301-8.

16. Secretaría de Salud. Norma Oficial Mexicana NOM-174-SSA1-1998, para el manejo integral de la obesidad. México: Diario Oficial de la Federación; 2000.

17. Ignacio de Ulíbarri J, González-Madroño A, de Villar NG, González P González B, Mancha A, et al. CONUT: a tool for controlling nutritional status. First validation in a hospital population. Nutr Hosp. 2005;20:38-45.

18. López Martínez J, Sánchez Castilla M, Ordóñez González FJ, Temprano Vázquez S, García de Lorenzo A, del Nogal Sáez F. [The usefulness of cholesterol as a nutritional-metabolic marker in the septic patient]. Nutr Hosp. 1995;10:24-31. 